\title{
Propagation of Love-Type Wave in Porous Medium over an Orthotropic Semi-Infinite Medium with Rectangular Irregularity
}

\author{
Pramod Kumar Vaishnav, Santimoy Kundu, Shishir Gupta, and Anup Saha \\ Department of Applied Mathematics, Indian School of Mines, Dhanbad 826004, India \\ Correspondence should be addressed to Pramod Kumar Vaishnav; pvaishnav.ism@gmail.com
}

Received 4 November 2015; Revised 12 January 2016; Accepted 14 January 2016

Academic Editor: Evangelos J. Sapountzakis

Copyright (c) 2016 Pramod Kumar Vaishnav et al. This is an open access article distributed under the Creative Commons Attribution License, which permits unrestricted use, distribution, and reproduction in any medium, provided the original work is properly cited.

\begin{abstract}
Propagation of Love-type wave in an initially stressed porous medium over a semi-infinite orthotropic medium with the irregular interface has been studied. The method of separation of variables has been adopted to get the dispersion relation of Love-type wave. The irregularity is assumed to be rectangular at the interface of the layer and half-space. Finally, the dispersion relation of Love wave has been obtained in classical form. The presence of porosity, irregularity, and initial stress in the dispersion equation approves the significant effect of these parameters in the propagation of Love-type waves in porous medium bounded below by an orthotropic half-space. The scientific effect of porosity, irregularity, and initial stress in the phase velocity of the Love-type wave propagation has been studied and shown graphically.
\end{abstract}

\section{Introduction}

The Earth contains fluid-saturated porous rocks on or below its surface in the form of sandstone and other sediments permeated by groundwater or oil; the diffusion of fluid and readjustment of fluid pressure have been acting as a triggering mechanism for earthquakes. So, the study of wave propagation in a porous medium has gained prime interest. The propagation of Love-type wave in porous media with irregular boundary surfaces is important leading to better understanding and prediction of behaviour of seismic wave at mountain roots, continental margins, and so forth. Love-type wave propagation in layered media has long been a research subject because of its practical importance in exploration of oil, geophysics, earthquake engineering, and underground water. The current work is concerned with the propagation of Love-type waves in initially stressed porous layer overlying semi-infinite orthotropic medium with irregular interface. It has been noticed that the presence of porosity, irregularity, and initial stress in the dispersion equation approves the significant effect of these parameters in the propagation of Love-type waves.

The intended applications of this theory may be found in the field of geophysics and the manufactured porous solids.
Various problems of waves and vibrations based on these theories of elasticity have been attempted by the researchers and have appeared in the open literature. Following Biot ([14]), the frequency equation has been used from the dynamic theory of wave propagation in fluid-saturated porous media. The effect of porosity, initial stress, and gravity has been described by many researchers in several Earth structures as when the porosity of the porous half-space increases, the phase velocity decreases, whereas the sandy parameter has increasing effect in the propagation of Love waves concluded by Pal and Ghorai [5]. Abo-Dahab et al. [6] discussed the rotation and magnetic field effect on surface waves propagation in an elastic layer lying over a generalized thermoelastic diffusive half-space with imperfect boundary. Ahmed and Abo-Dahab [7] pointed out the propagation of Love waves in an orthotropic granular layer under initial stress overlying a semi-infinite granular medium. Chattaraj and Samal [8] discussed the effect of gravity, porosity in the Love waves in the fibre-reinforced layer over a gravitating porous halfspace. Chen et al. [9] discussed a mixture theory analysis for the surface wave propagation in an unsaturated porous medium. Kalyani et al. [10] pointed out the finite difference modeling of seismic wave propagation in monoclinic media. 
Ghorai et al. [11] considered the Love waves in a fluidsaturated porous layer under a rigid boundary and lying over an elastic half-space under gravity. Chattopadhyay et al. [12] concluded the effect of point source and heterogeneity on the propagation of $\mathrm{SH}$ waves in a viscoelastic layer over a viscoelastic half-space. Gupta et al. [13] established the possibility of Love wave propagation in a porous layer under the effect of linearly varying directional rigidities. Gupta et al. [14] provided the effect of initial stress on propagation of Love waves in an anisotropic porous layer. Ke et al. [15] discussed the propagation of Love waves in an inhomogeneous fluid-saturated porous layered halfspace with properties varying exponentially. Kończak [16] displayed the propagation of Love waves in a fluid-saturated porous anisotropic layer. Analysis of wave motion at the boundary surface of orthotropic thermoelastic material with voids and isotropic elastic half-space was studied by R. Kumar and R. Kumar [17]. Liu and Boer [18] explained the dispersion and attenuation of surface waves in a fluid-saturated porous medium. Chakraborty and Dey [19] discussed the propagation of Love waves in water-saturated soil underlain by a heterogeneous elastic medium. Sharma [20] investigated the wave propagation in a general anisotropic poroelastic medium with anisotropic permeability: phase velocity and attenuation. Wang and Zhang [21] discussed the propagation of Love waves in a transversely isotropic fluid-saturated porous layered half-space. Abd-Alla et al. ([22-26]) pointed out the propagation of Love wave, Rayleigh wave in various structures of the Earth. The authors used distinct medium of the Earth to propagate the seismic waves, such as orthotropic, magnetoelastic, fibre-reinforced anisotropic, viscoelastic, and magnetothermoelastic medium. In such a medium, they concluded the effect of various parameters such as rotation and transmission, influence of initial stress on orthotropic medium, and magnetic and gravity field on the propagation of seismic waves. Abd-alla and Abo-dahab [27] investigated the Rayleigh waves in magnetothermoviscoelastic solid with thermal relaxation times. Abo-Dahab et al. [28] concluded the effect of magnetism and rotation on surface waves in fibre-reinforced anisotropic general viscoelastic media of higher order.

In this paper, we use the porous medium (layer) over an orthotropic half-space with the effect of irregular interface in the propagation of Love-type waves. The main attention is paid to the influence of irregularity of interface, porosity, and initial stress on the propagation of Love-type waves in porous-orthotropic medium (Figure 1). The rectangular irregularity at the interface of layered half-space affected the phase velocity of Love-type waves. The classical dispersion relation of Love wave has been obtained in particular cases as Love [29]. To study the effect of porosity, initial stress, and irregularity, we represent numerical data from Gubbins [30]. The study shows that the irregularity and porosity and initial stress have significant effect on the phase velocity of Lovetype wave. For graphical representation, MATLAB software has been used to generate results. The study of surface wave propagation with irregular interface helps civil engineers in building construction, analysis of earthquake in mountain

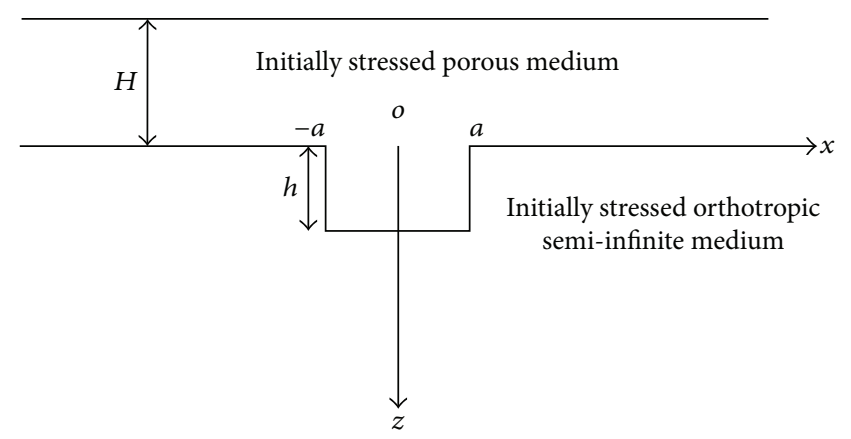

FIGURE 1: Geometry of the problem.

roots, continental margins, and so forth. It is also useful for the study of seismic waves generated by artificial explosions.

\section{Mathematical Formulation of the Problem}

We have considered a model consisting of initially stressed porous layer of finite thickness $H$ overlying an orthotropic half-space with irregular interface. The rectangular irregular surface has been taken at the interface of the layered-halfspace model with length $2 a$ and depth $h$. $x$-axis is parallel to the direction of wave propagation, and $z$-axis is vertically downward to the direction of wave propagation. The upper surface of the porous layer is stress-free. The shape of the irregularity at the interface of the porous layer is taken as $z=\epsilon f(x)$, where

$$
\begin{gathered}
f(x)= \begin{cases}0 ; & |x|>a, \\
2 a ; & |x| \leq a,\end{cases} \\
\epsilon=\frac{h}{2 a} \ll 1 .
\end{gathered}
$$

\section{Solution of the Initially Stressed Porous Layer}

Neglecting the viscosity, in the absence of body forces, the dynamical equations of motion for initially stressed anisotropic porous medium can be written as Biot [4]:

$$
\begin{gathered}
\frac{\partial s_{11}}{\partial x}+\frac{\partial s_{12}}{\partial y}+\frac{\partial s_{13}}{\partial z}-P_{1}\left(\frac{\partial w_{z}}{\partial y}-\frac{\partial w_{y}}{\partial z}\right) \\
=\frac{\partial^{2}}{\partial t^{2}}\left(\rho_{11} u_{1}+\rho_{12} U_{x}\right), \\
\frac{\partial s_{21}}{\partial x}+\frac{\partial s_{22}}{\partial y}+\frac{\partial s_{23}}{\partial z}-P_{1}\left(\frac{\partial w_{z}}{\partial x}\right) \\
=\frac{\partial^{2}}{\partial t^{2}}\left(\rho_{11} v_{1}+\rho_{12} V_{y}\right),
\end{gathered}
$$




$$
\begin{gathered}
\frac{\partial s_{31}}{\partial x}+\frac{\partial s_{32}}{\partial y}+\frac{\partial s_{33}}{\partial z}-P_{1}\left(\frac{\partial w_{y}}{\partial x}\right) \\
=\frac{\partial^{2}}{\partial t^{2}}\left(\rho_{11} w_{1}+\rho_{12} W_{z}\right), \\
\frac{\partial s^{\prime}}{\partial x}=\frac{\partial^{2}}{\partial t^{2}}\left(\rho_{11} u_{1}+\rho_{22} U_{x}\right), \\
\frac{\partial s^{\prime}}{\partial y}=\frac{\partial^{2}}{\partial t^{2}}\left(\rho_{11} v_{1}+\rho_{22} V_{y}\right), \\
\frac{\partial s^{\prime}}{\partial z}=\frac{\partial^{2}}{\partial t^{2}}\left(\rho_{11} w_{1}+\rho_{22} W_{z}\right),
\end{gathered}
$$

where $\left(u_{1}, v_{1}, w_{1}\right)$ and $\left(U_{x}, V_{y}, W_{z}\right)$ are displacement components of solid and liquid part of porous medium in $x, y$, and $z$ direction, respectively, and $P_{1}$ represents the initial stress in porous medium. The incremental stress components of solid part of porous medium are $s_{i j}(i, j=1,2,3)$ and $s^{\prime}$ is the stress vector of liquid part of porous medium, where $s^{\prime}=-f p, f$ is the porosity, and $p$ is the fluid pressure, and $w_{x}, w_{y}$, and $w_{z}$ are angular components, defined as

$$
\begin{aligned}
& w_{x}=\frac{1}{2}\left(\frac{\partial w_{1}}{\partial y}-\frac{\partial v_{1}}{\partial z}\right), \\
& w_{y}=\frac{1}{2}\left(\frac{\partial u_{1}}{\partial z}-\frac{\partial w_{1}}{\partial x}\right), \\
& w_{z}=\frac{1}{2}\left(\frac{\partial v_{1}}{\partial x}-\frac{\partial u_{1}}{\partial y}\right) .
\end{aligned}
$$

The relations between mass coefficients $\rho_{11}, \rho_{12}$, and $\rho_{22}$ and the densities $\rho^{\prime}, \rho_{s}$, and $\rho_{w}$ of the layer in solid and liquid porous media are given by

$$
\begin{aligned}
& \rho_{11}+\rho_{12}=(1-f) \rho_{s}, \\
& \rho_{12}+\rho_{22}=f \rho_{w},
\end{aligned}
$$

and the mass density of the bulk material is

$$
\rho^{\prime}=\rho_{11}+\rho_{22}+2 \rho_{12}=\rho_{s}+f\left(\rho_{w}-\rho_{s}\right) \text {. }
$$

These mass coefficients also satisfy the following inequalities:

$$
\begin{aligned}
\rho_{11} & >0, \\
\rho_{22} & >0, \\
\rho_{12} & <0, \\
\rho_{11} \rho_{12}-\rho_{12}^{2} & >0 .
\end{aligned}
$$

In the water-saturated anisotropic porous medium, the stressstrain relations are

$$
\begin{aligned}
s_{11}= & \left(A+P_{1}\right) e_{x x}+\left(A-2 N+P_{1}\right) e_{y y}+\left(F+P_{1}\right) e_{z z} \\
& +Q \epsilon, \\
s_{22}= & (A-2 N) e_{x x}+A e_{y y}+F e_{z z}+Q \epsilon, \\
s_{33}= & F e_{x x}+F e_{y y}+C e_{z z}+Q \epsilon, \\
s_{12}= & 2 N e_{x y}, \\
s_{23}= & 2 L e_{y z}, \\
s_{13}= & 2 L e_{z x}, \\
e_{i j}= & \frac{1}{2}\left(\frac{\partial u_{i}}{\partial x_{j}}+\frac{\partial u_{j}}{\partial x_{i}}\right), \\
\epsilon= & \frac{\partial U_{x}}{\partial x}+\frac{\partial U_{y}}{\partial y}+\frac{\partial U_{x}}{\partial z},
\end{aligned}
$$

where $N$ and $L$ represent the shear moduli of the anisotropic layer in the $x$ and $z$ direction, respectively, whereas $A, F$, and $C$ are elastic constants for the medium. The positive quantity $Q$ is the measure of coupling between the changes of volume of solid and liquid.

For the propagation of Love waves along the $x$ direction,

$$
\begin{aligned}
& u_{1}=0, \\
& w_{1}=0, \\
& v_{1}=v_{1}(x, x, t), \\
& U_{x}=0, \\
& W_{z}=0, \\
& V_{y}=V(x, z, t) .
\end{aligned}
$$

Thus, the stress-strain relations are

$$
\begin{aligned}
& s_{23}=2 L e_{y z}, \\
& s_{12}=2 N e_{x y} .
\end{aligned}
$$

Using (10) in (2), the equations of motion which are not automatically satisfied are

$$
\begin{gathered}
\frac{\partial s_{21}}{\partial x}+\frac{\partial s_{22}}{\partial y}+\frac{\partial s_{23}}{\partial z}-P_{1}\left(\frac{\partial w_{z}}{\partial x}\right) \\
=\frac{\partial^{2}}{\partial t^{2}}\left(\rho_{11} v_{1}+\rho_{12} V_{y}\right), \\
\frac{\partial s}{\partial y}=0=\frac{\partial^{2}}{\partial t^{2}}\left(\rho_{12} v_{1}+\rho_{22} V_{y}\right) .
\end{gathered}
$$


By using (9) and (10) with Love wave condition, the above equations reduced into

$$
\begin{aligned}
\left(N-\frac{P_{1}}{2}\right) \frac{\partial^{2} v_{1}}{\partial x^{2}}+L \frac{\partial^{2} v_{1}}{\partial z^{2}} & =\frac{\partial^{2}}{\partial t^{2}}\left(\rho_{11} v_{1}+\rho_{12} V\right), \\
\frac{\partial^{2}}{\partial t^{2}}\left(\rho_{12} v_{1}+\rho_{22} V\right) & =0 .
\end{aligned}
$$

From $\left(\partial^{2} / \partial t^{2}\right)\left(\rho_{12} v_{1}+\rho_{22} V\right)=0$ and $\rho_{12} v_{1}+\rho_{22} V=d^{\prime \prime}$ (say) $V=\left(d^{\prime \prime}-\rho_{12} v_{1}\right) / \rho_{22}$. Now, $\left(\partial^{2} / \partial t^{2}\right)\left(\rho_{11} v_{1}+\rho_{12} V\right)=$ $d^{\prime}\left(\partial^{2} v_{1} / \partial t^{2}\right)$, where $d^{\prime}=\rho_{11}-\rho_{12}^{2} / \rho_{22}$.

Therefore, (12) can be written as

$$
\left(N-\frac{P_{1}}{2}\right) \frac{\partial^{2} v_{1}}{\partial x^{2}}+L \frac{\partial^{2} v_{1}}{\partial z^{2}}=d^{\prime} \frac{\partial^{2} v_{1}}{\partial t^{2}} .
$$

From the above equation, the shear wave velocity along the $x$ direction is $\sqrt{\left(N-P_{1} / 2\right) / d^{\prime}}$ and along the $z$ direction is $\sqrt{L / d^{\prime}}$.

In the anisotropic porous medium, the shear wave velocity along the $x$ direction can be expressed as

$$
\beta=\sqrt{\frac{N-P_{1} / 2}{d^{\prime}}}=\beta_{1} \sqrt{\frac{1-\zeta^{\prime}}{d_{1}}},
$$

where $d_{1}=\gamma_{11}-\gamma_{12}^{2} / \gamma_{22}, \beta_{1}=\sqrt{N / \rho^{\prime}}, \beta_{1}$ is the shear wave velocity in the corresponding initial stress-free, nonporous, anisotropic, elastic medium along the $x$ direction, $\zeta^{\prime}=P_{1} / 2 \mathrm{~N}$ is the nondimensional parameter due to the initial stress $P_{1}$, and

$$
\begin{aligned}
& \gamma_{11}=\frac{\rho_{11}}{\rho^{\prime}}, \\
& \gamma_{13}=\frac{\rho_{13}}{\rho^{\prime}}, \\
& \gamma_{23}=\frac{\rho_{23}}{\rho^{\prime}}
\end{aligned}
$$

are the dimensionless parameters for the materials of the porous layer as obtained by Biot [3].

Thus, one gets the following:

(i) $d_{1} \rightarrow 1$, when the layer is nonporous solid.

(ii) $d_{1} \rightarrow 0$, when the layer is fluid.

(iii) $0<d_{1}<1$, when the layer is poroelastic.

Assume the solution of (14) as

$$
v_{1}(x, z, t)=V_{1}(z) e^{i k(x-c t)} .
$$

Substituting (17) into (14), we obtain

$$
\frac{d^{2} V_{1}}{d z^{2}}+m_{1}^{2} V_{1}=0
$$

where $m_{1}=k \sqrt{(1 / L)\left(c^{2} d^{\prime}-N+P_{1} / 2\right)}$.
Therefore, the solution of (18) takes the form $V_{1}(z)=$ $A_{1} \cos \left\{m_{1} z\right\}+A_{2} \sin \left\{m_{1} z\right\}$, where $A_{1}$ and $A_{2}$ are arbitrary constants. Hence, the displacement in the porous layer is given by

$$
v_{1}=\left[A_{1} \cos \left\{m_{1} z\right\}+A_{2} \sin \left\{m_{1} z\right\}\right] e^{i k(x-c t)} .
$$

This is the displacement on an initially stressed anisotropic porous layer, where

$$
\begin{aligned}
m_{1} & =k \sqrt{\frac{\left[c^{2} d^{\prime}-\left(N-P_{1} / 2\right)\right]}{L}} \\
& =k \sqrt{\gamma d_{1}\left[\frac{c^{2}}{\beta_{1}^{2}}-\frac{1-\zeta^{\prime}}{d_{1}}\right]},
\end{aligned}
$$

$\gamma=N / L, \zeta^{\prime}=P_{1} / 2 N, \beta_{1}^{2}=N / \rho^{\prime}$, and $k$ is the wave number.

\section{Solution of Orthotropic Half-Space}

The equations of motion for the orthotropic medium under initial stress in the absence of body forces are

$$
\begin{aligned}
\frac{\partial \tau_{11}}{\partial x}+\frac{\partial \tau_{12}}{\partial y}+\frac{\partial \tau_{13}}{\partial z}-P_{2}\left(\frac{\partial w_{z}^{\prime}}{\partial y}-\frac{\partial w_{y}^{\prime}}{\partial z}\right) & =\rho_{2} \frac{\partial^{2} u_{2}}{\partial t^{2}} \\
\frac{\partial \tau_{21}}{\partial x}+\frac{\partial \tau_{22}}{\partial y}+\frac{\partial \tau_{23}}{\partial z}-P_{2} \frac{\partial w_{z}^{\prime}}{\partial x} & =\rho_{2} \frac{\partial^{2} v_{2}}{\partial t^{2}} \\
\frac{\partial \tau_{31}}{\partial x}+\frac{\partial \tau_{32}}{\partial y}+\frac{\partial \tau_{33}}{\partial z}-P_{2} \frac{\partial w_{y}^{\prime}}{\partial x} & =\rho_{2} \frac{\partial^{2} w_{2}}{\partial t^{2}}
\end{aligned}
$$

where $u_{2}, v_{2}$, and $w_{2}$ are the displacement components in the orthotropic medium and $w_{x}^{\prime}, w_{y}^{\prime}$, and $w_{z}^{\prime}$ are the rotational components along $x, y$, and $z$ direction. Here, $\tau_{i j}$ are the incremental stress components and $\rho_{2}$ is the density of the material in the semi-infinite medium.

The stress-strain relations in the orthotropic medium are

$$
\begin{aligned}
& \tau_{11}=B_{11} e_{11}+B_{12} e_{22}+B_{13} e_{33}, \\
& \tau_{12}=2 Q_{3} e_{12}, \\
& \tau_{22}=B_{21} e_{11}+B_{22} e_{22}+B_{23} e_{33}, \\
& \tau_{23}=2 Q_{1} e_{23}, \\
& \tau_{33}=B_{31} e_{11}+B_{32} e_{22}+B_{33} e_{33}, \\
& \tau_{31}=2 Q_{2} e_{31},
\end{aligned}
$$

where $B_{i j}$ are the incremental normal elastic coefficient and $Q_{i}$ are shear moduli, whereas $e_{i j}$ are the strain components. 
Again, using the Love waves conditions $u_{2}=0, w_{2}=0$, and $v_{2}=v_{2}(x, z, t)$, the only equation of motion from (21) and (22) for the orthotropic half-space can be written as

$$
\begin{aligned}
& \frac{\partial}{\partial x}\left(Q_{3} \frac{\partial v_{2}}{\partial x}\right)+\frac{\partial}{\partial z}\left(Q_{1} \frac{\partial v_{2}}{\partial z}\right)-P_{2} \frac{\partial}{\partial x}\left(\frac{1}{2} \frac{\partial v_{2}}{\partial x}\right) \\
& \quad=\rho_{2} \frac{\partial^{2} v_{2}}{\partial t^{2}} \\
& \left(Q_{3}-\frac{P_{2}}{2}\right) \frac{\partial^{2} v_{2}}{\partial x^{2}}+Q_{1} \frac{\partial^{2} v_{2}}{\partial z^{2}}=\rho_{2} \frac{\partial^{2} v_{2}}{\partial t^{2}},
\end{aligned}
$$

and the stress components $\tau_{12}=2 Q_{3} e_{12}$ and $\tau_{23}=2 Q_{1} e_{23}$; other components will be zero.

For wave propagation along $x$ direction, it may be assumed that $v_{2}=V_{2}(z) e^{i k(x-c t)}$, where $k$ is the wave number and $c$ is the phase velocity; then (23) can be reduced:

$$
\frac{d^{2} V_{2}}{d z^{2}}-m_{2}^{2} V_{2}=0
$$

where $m_{2}^{2}=\left(k^{2} / Q_{1}\right)\left[\left(Q_{3}-P_{2} / 2\right)-c^{2} \rho_{2}\right]$. So, the solution for the initially stressed semi-infinite orthotropic medium will be of the form

$$
v_{2}=A_{3} e^{-m_{2} z} e^{i k(x-c t)},
$$

where $A_{3}$ is the arbitrary constant.

\section{Boundary Conditions}

The upper surface of the porous layer is stress-free; that is,

$$
L \frac{\partial v_{1}}{\partial z}=0, \quad \text { at } z=-H,
$$

at the irregular interface; that is, $z=\epsilon f(x)$,

$$
\begin{aligned}
& v_{1}(z)=v_{2}(z), \\
& L \frac{\partial v_{1}}{\partial z}=Q_{1} \frac{\partial v_{2}}{\partial z} .
\end{aligned}
$$

Now, applying the boundary conditions, we have

$$
\begin{aligned}
& \sin \left\{m_{1} H\right\} A_{1}+\cos \left\{m_{1} H\right\} A_{2}=0, \\
& \cos m_{1}(\epsilon f(x))+A_{2} \sin m_{1}(\epsilon f(x))=A_{3} e^{-m_{2} \epsilon f(x)}, \\
& L\left\{-m_{1} A_{1} \sin m_{1}(\epsilon f(x))+m_{1} A_{2} \cos m_{1}(\epsilon f(x))\right\} \\
& \quad=-m_{2} Q_{1} e^{-m_{2} \epsilon f(x)} A_{3} .
\end{aligned}
$$

The generalized dispersion equation of Love-type wave will be obtained by eliminating arbitrary constants from the above equations as

$$
\left|\begin{array}{ccc}
\sin m_{1} H & \cos m_{1} H & 0 \\
\cos m_{1}(\epsilon f(x)) & \sin m_{1}(\epsilon f(x)) & -e^{-m_{2} \epsilon f(x)} \\
-L m_{1} \sin m_{1}(\epsilon f(x)) & L m_{1} \cos m_{1}(\epsilon f(x)) & Q_{1} m_{2} e^{-m_{2} \epsilon f(x)}
\end{array}\right|
$$$$
=0 \text {, }
$$

or

$$
\begin{aligned}
\sin & m_{1} H \\
\cdot & \left\{Q_{1} m_{2} \sin m_{1}(\epsilon f(x))+L m_{1} \cos m_{1}(\epsilon f(x))\right\} \\
- & \cos m_{1} H \\
\cdot & \left\{Q_{1} m_{2} \cos m_{1}(\epsilon f(x))-L m_{1} \sin m_{1}(\epsilon f(x))\right\} \\
= & 0 .
\end{aligned}
$$

The generalized dispersion relation of Love wave has been obtained as

$$
\tan m_{1} H=\frac{\left\{Q_{1} m_{2} \cos \left(m_{1} h\right)-L m_{1} \sin \left(m_{1} h\right)\right\}}{\left\{Q_{1} m_{2} \sin \left(m_{1} h\right)+L m_{1} \cos \left(m_{1} h\right)\right\}} .
$$

\section{Particular Cases}

Case 1. In case the porous layer has no irregularity, that is, $h=0$, (32) reduces to

$$
\begin{aligned}
& \tan k H \sqrt{\gamma d_{1}\left[\frac{c^{2}}{\beta_{1}^{2}}-\frac{1-\zeta^{\prime}}{d_{1}}\right]} \\
& =\frac{Q_{1}}{L} \frac{\sqrt{\left[\left(Q_{3} / Q_{1}-P / 2 Q_{1}\right)-c^{2} / \beta_{2}^{2}\right]}}{\sqrt{\gamma d_{1}\left[c^{2} / \beta_{1}^{2}-\left(1-\zeta^{\prime}\right) / d_{1}\right]}},
\end{aligned}
$$

where $\beta_{2}=\sqrt{Q_{1} / \rho_{2}}$, which is the dispersion relation of Love-type wave when the interface of the layered half-space is regular.

Case 2. For the nonporous homogeneous layer $d_{1}=1, N=$ $L=\mu_{1}, \gamma=1, P_{1} / 2 \mu_{1}=0$, (33) becomes

$$
\begin{aligned}
\tan & \left\{k H \sqrt{\frac{c^{2}}{\beta_{1}^{2}}-1}\right\} \\
& =\frac{Q_{1}}{\mu_{1}} \frac{\sqrt{\left[\left(Q_{3} / Q_{1}-P / 2 Q_{1}\right)-c^{2} / \beta_{2}^{2}\right]}}{\sqrt{c^{2} / \beta_{1}^{2}-1}} .
\end{aligned}
$$

The above equation represents the dispersion equation of Love-type wave for initial stress-free nonporous homogeneous layer.

Case 3. When the semi-infinite medium is initially stress-free and homogeneous with rigidity $\mu_{2}$ (i.e., $Q_{1} \rightarrow Q_{2} \rightarrow \mu_{2}$ and $P_{2} / 2 \mu_{2}=0$ ), (34) becomes

$$
\tan \left\{k H \sqrt{\left(\frac{c^{2}}{\beta_{1}^{2}}\right)-1}\right\}=\frac{\mu_{2}}{\mu_{1}} \frac{\sqrt{1-c^{2} / \beta_{2}^{2}}}{\sqrt{c^{2} / \beta_{1}^{2}-1}}
$$

which is the classical dispersion relation of Love wave (as Love [29]) in a homogeneous layer over a homogeneous half-space. 


\section{Numerical Calculations and Discussions}

Based on dispersion (32), numerical results are provided to show the propagation characteristics of Love waves in an initially stressed anisotropic porous layer over an orthotropic half-space. The effect of porosity, initial stress, and irregularity of the porous layer in phase velocity $c / \beta_{1}$ has been analyzed graphically.

To study the effect of porosity, initial stress, and irregularity, we represent the numerical data from Gubbins [30] as follows:

(a) For the orthotropic half-space,

$$
\begin{aligned}
& Q_{1}=5.82 \times 10^{10} \mathrm{~N} / \mathrm{m}^{2}, \\
& Q_{3}=3.99 \times 10^{10} \mathrm{~N} / \mathrm{m}^{2}, \\
& \rho_{2}=4.5 \times 10^{3} \mathrm{~kg} / \mathrm{m}^{3} .
\end{aligned}
$$

(b) For the anisotropic porous layer,

$$
\begin{aligned}
L & =0.1387 \times 10^{10} \mathrm{~N} / \mathrm{m}^{2}, \\
N & =0.2774 \times 10^{10} \mathrm{~N} / \mathrm{m}^{2}, \\
\rho_{11} & =1.926137 \times 10^{3} \mathrm{~kg} / \mathrm{m}^{3}, \\
\rho_{12} & =-0.002137 \times 10^{3} \mathrm{~kg} / \mathrm{m}^{3}, \\
\rho_{22} & =0.215337 \times 10^{3} \mathrm{~kg} / \mathrm{m}^{3}, \\
f & =0.26 .
\end{aligned}
$$

In all the figures, curves have been plotted as phase velocity $c / \beta_{1}$ along vertical axis against dimensionless wave number $k H$ along horizontal axis. It has been observed that the maximum changes happen in phase velocity between $k H=0.1$ and $k H=1.0$. The phase velocity of Love-type wave affected by the porosity of the medium, initial stress, and irregular interface of the layer and half-space and the significant impact of the abovementioned parameters has been shown in the figures.

Figure 2 shows the effect of height of irregularity $(h / H)$ on the phase velocity of Love-type wave in anisotropic porous medium. The presence of irregularity at the interface of layer and half-space has the significant impact on the propagation of Love-type wave; the height $(h / H)$ of irregular surface has been taken as $0.1,0.2$, and 0.3 for curves 1,2 , and 3 , respectively, whereas $P_{1} / 2 \mu_{1}=0.2, P_{2} / 2 \mu_{2}=0.3$, and $d_{1}=$ 0.01 are constants. It is observed that the phase velocity $c / \beta_{1}$ of Love-type waves decreases as the height of irregularity increases; that is, the speed of a Love-type wave depends on the height of irregularity in porous medium and the obtained result may be helpful for civil construction and evaluation of earthquake damage in mountain region.

Figure 3 depicts the influence of initial stress $P_{1} / 2 \mu_{1}$ associated with porous layer on the phase velocity $c / \beta_{1}$ of Love-type wave. Curve 1, curve 2, and curve 3 demonstrate the impact of initial stress on the phase velocity of a Lovetype wave for $P_{1} / 2 \mu_{1}=0.1,0.2$, and 0.3 , respectively. It is

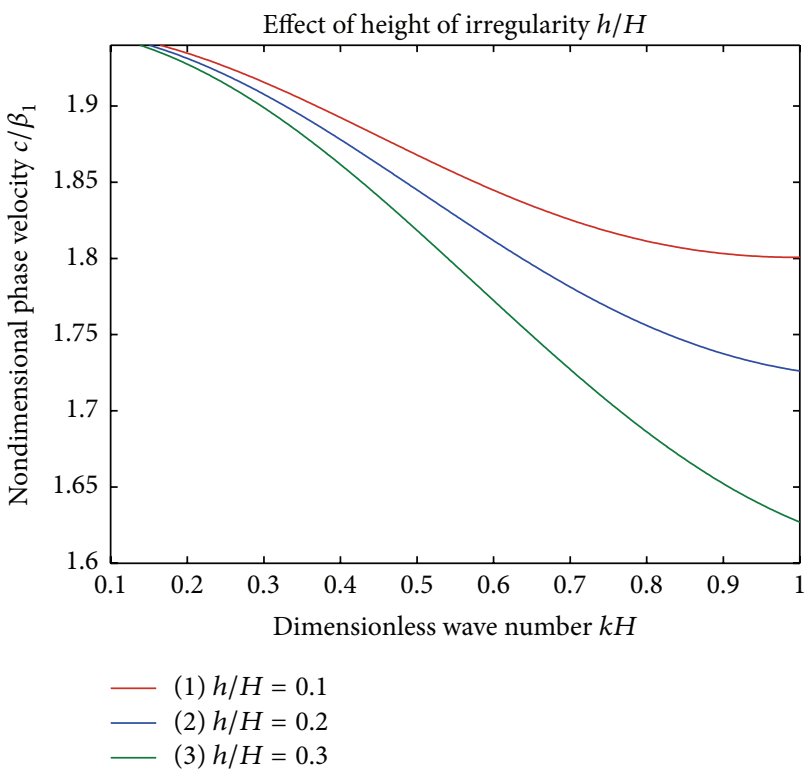

FIgURE 2: Variation of phase velocity $\left(c / \beta_{1}\right)$ with the wave number $(k H)$ for different values of $h / H(h / H=0.1,0.2,0.3)$ when $P_{1} / 2 \mu_{1}=$ $0.2, P_{2} / 2 \mu_{2}=0.3, d_{1}=0.01$, and $\gamma=1$.

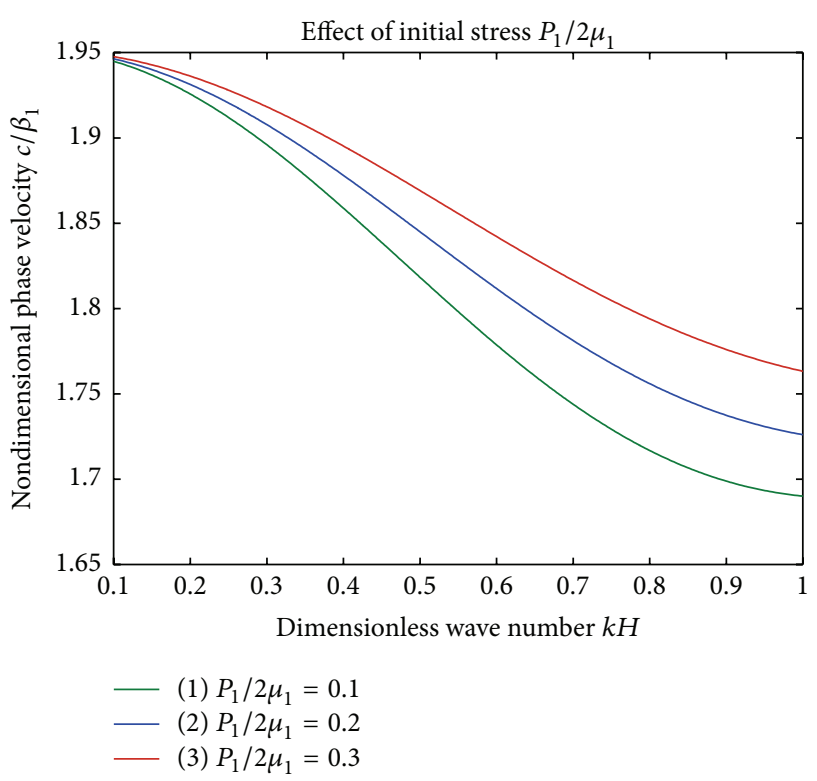

FIGURE 3: Variation of phase velocity $\left(c / \beta_{1}\right)$ with the wave number $(k H)$ for different values of $P_{1} / 2 \mu_{1}\left(P_{1} / 2 \mu_{1}=0.1,0.2,0.3\right)$ when $h / H=0.1, P_{2} / 2 \mu_{2}=0.3, d_{1}=0.01$, and $\gamma=1$.

observed that the presence of initial stress in porous medium increases the phase velocity of Love-type wave as the value of initial stress increases. It is also observed that the behaviour of Love wave speed in initially stressed porous medium and at irregular interface is different, so the term irregularity in the Earth plays an important role in the propagation of surface waves.

Figure 4 shows the effect of initial stress $P_{2} / 2 \mu_{2}$ associated with half-space on the phase velocity of Love-type waves 


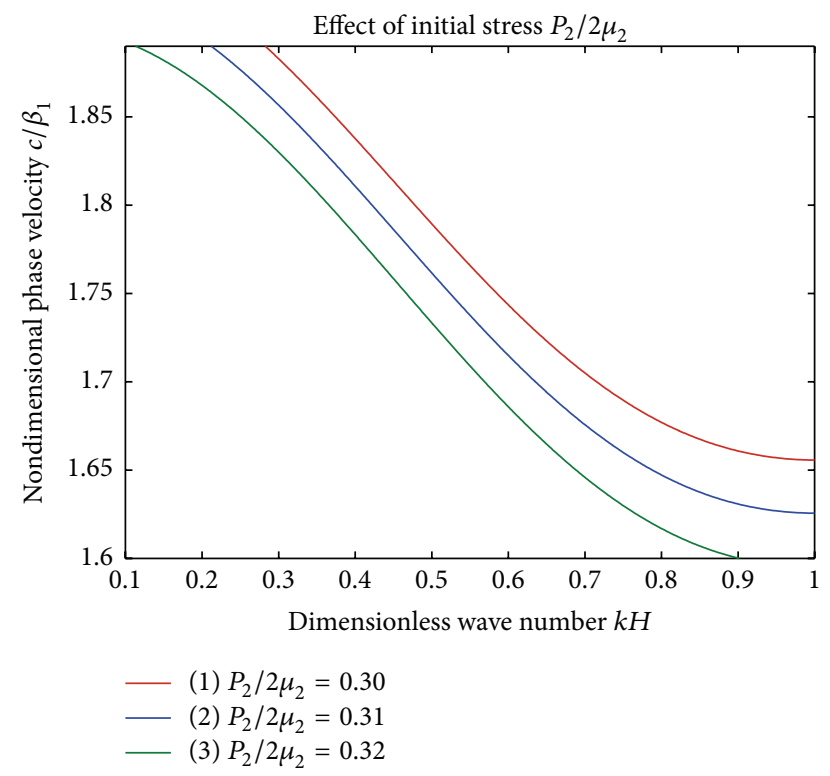

FIGURE 4: Variation of phase velocity $\left(c / \beta_{1}\right)$ with the wave number $(k H)$ for different values of $P_{2} / 2 \mu_{2}\left(P_{2} / 2 \mu_{2}=0.30,0.31,0.32\right)$ when $h / H=0.1, P_{1} / 2 \mu_{1}=0.2, d_{1}=0.01$, and $\gamma=1$.

in porous medium. The presence of initial stress in the orthotropic medium affected the phase velocity of Lovetype wave significantly. Curve 1, curve 2, and curve 3 have been plotted for $P_{2} / 2 \mu_{2}=0.30$ and $P_{2} / 2 \mu_{2}=0.31$ and 0.32 in the presence of irregular interface. It is found that the phase velocity decreases as the value of initial stress increases and has much dominance at large values of wave number. The presence of initial stress $P_{1} / 2 \mu_{1}$ in the porous medium increases the phase velocity of Love-type wave, whereas the phase velocity decreases in the presence of initial stress $\left(P_{2} / 2 \mu_{2}\right)$ in orthotropic medium. It is observed that the presence of irregularity of the interface affected the phase velocity of Love wave in different ways in both mediums.

Figure 5 pointed out the influence of porosity $\left(d_{1}\right)$ of the medium on the phase velocity of Love-type wave. The porosity is taken as $d_{1}=0.01,0.02$, and 0.03 for curve 1 , curve 2 , and curve 3 , respectively. The curves apart from each other between $k H=0.1$ and 1.0 show that $d_{1}$ has a perfect influence over the phase velocity of Love-type wave. It is observed that the phase velocity increases rapidly as the value of porosity increases. It has been found that, with the increase in wave number, the phase velocity decreases rapidly in each of these figures under the considered values of various parameters.

Figure 6 described the impact of height of irregularity in the absence of initial stress $\left(P_{1} / 2 \mu_{1}\right)$ on the phase velocity of Love-type wave. It has been observed that the phase velocity decreases with the depth of irregularity in an orthotropic medium.

The study of seismic waves gives important information about the layered Earth structure and has been used to determine the epicenter of the earthquake. Seismologists are able to learn about the Earth's internal structure by measuring the arrival of seismic waves at stations around the world because these waves travel at different speeds through

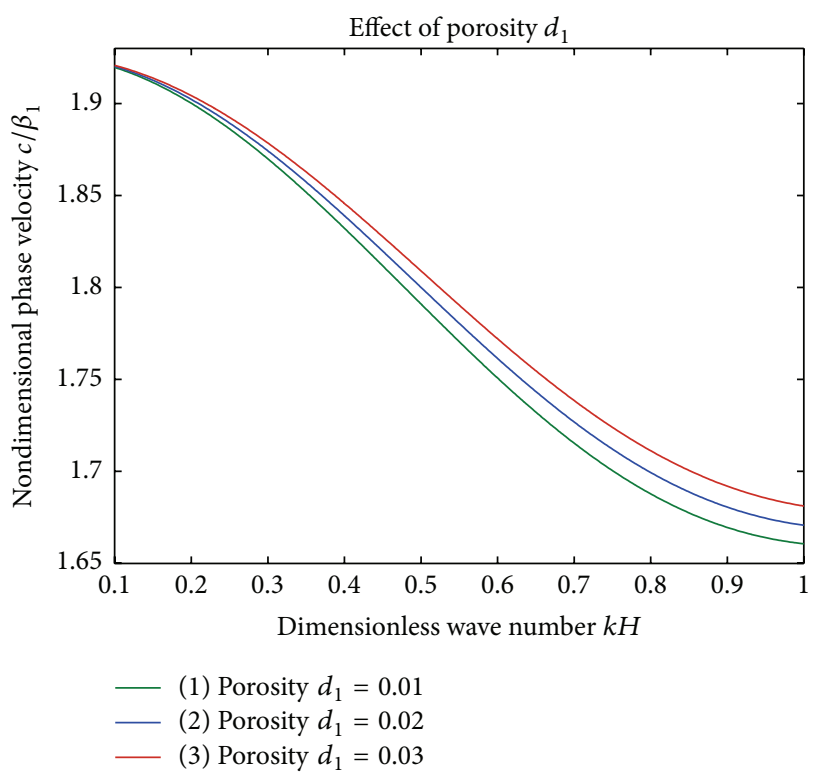

FIGURE 5: Variation of phase velocity $\left(c / \beta_{1}\right)$ with the wave number $(\mathrm{kH})$ for different values of porosity $\left(d_{1}=0.01,0.02,0.03\right)$ when $h / H=0.1, P_{1} / 2 \mu_{1}=0.1, P_{2} / 2 \mu_{2}=0.31$, and $\gamma=1$.

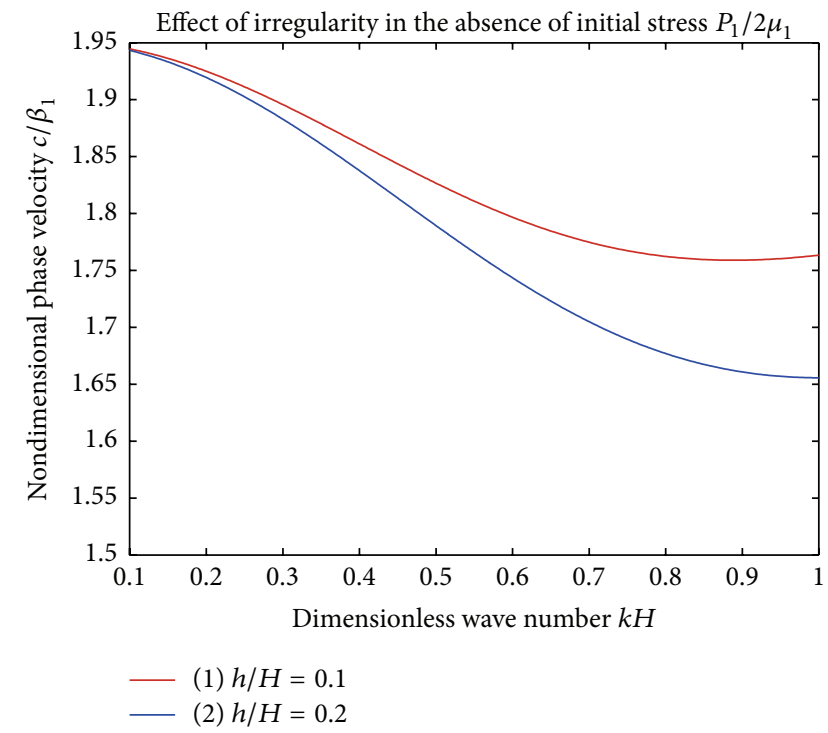

FIgURE 6: Variation of phase velocity $\left(c / \beta_{1}\right)$ with the wave number $(k H)$ for different values of $h / H(h / H=0.1,0.2)$ when $P_{1} / 2 \mu_{1}=0.0$, $P_{2} / 2 \mu_{2}=0.3, d_{1}=0.01$, and $\gamma=1$.

different materials. Knowing how fast these waves travel through the Earth, seismologists can calculate the time when the earthquake occurred and its location by comparing the times when shaking was recorded at several stations. If a wave arrives late, it passed through a hot, soft part of the Earth.

\section{Conclusions}

Propagation of Love-type waves in an initially stressed anisotropic porous layer over an initially stressed orthotropic 
medium with rectangular irregularity has been discussed. The method of separation of variables has been adopted to solve the equation of motion, separately, for different media using suitable boundary condition at the interface of anisotropic porous layer and orthotropic half-space with irregular interface. The dispersion relation of Love-type wave has been obtained and coincides with the classical dispersion relation of Love wave in particular cases. The presence of porosity, irregularity, and initial stress in the dispersion equation approves the significant effect of these parameters on the propagation of Love-type wave in porous medium bounded below by an orthotropic half-space. It has been observed that the maximum changes happen in phase velocity between $k H=0.1$ and $k H=1.0$. The conclusions are as follows:

(i) The height $h / H$ of the irregularity affected the phase velocity of Love-type wave, and the phase velocity $c / \beta_{1}$ decreases with increases in the height of the irregularity. It has been noticed that the rectangular irregularity of interface is more effective for high range of wave number $\mathrm{kH}$.

(ii) It is observed that the porosity also has a dominant role in the propagation of Love-type wave. When the porosity of the porous layer increases, the phase velocity of the Love wave also increases in such a structure.

(iii) The phase velocity increases with increases in initial stress $\left(P_{1} / 2 \mu_{1}\right)$ of the porous layer, whereas the phase velocity gradually decreases with increases in initial stress $\left(P_{2} / 2 \mu_{2}\right)$ of orthotropic half-space.

(iv) The height of irregularity has the impact on the phase velocity of Love-type wave in the absence of initial stress $\left(P_{1} / 2 \mu_{1}\right)$. It has been observed that the phase velocity decreases with the depth of irregularity in the orthotropic medium.

It is observed that the presence of porosity, initial stress, and irregularity affected the phase velocity of Love-type wave and has much dominance at large values of wave number. The initial stress in the porous medium increases the phase velocity of Love-type wave, whereas the phase velocity decreases in orthotropic medium due to initial stress. The phase velocity of Love-type wave also decreases with the depth of irregularity in an orthotropic medium.

\section{Conflict of Interests}

The authors declare that there is no conflict of interests regarding the publication of this paper.

\section{Acknowledgment}

The authors convey their sincere thanks to the Indian School of Mines, Dhanbad, India, for providing them with the best facilities.

\section{References}

[1] M. A. Biot, "Theory of propagation of elastic waves in a fluidsaturated porous solid. I. Low-frequency range," The Journal of the Acoustical Society of America, vol. 28, pp. 168-178, 1956.

[2] M. A. Biot, "Theory of propagation of elastic waves in a fluidsaturated porous solid. II. Higher frequency range," Acoustical Society of America. Journal, vol. 28, no. 2, pp. 179-191, 1956.

[3] M. A. Biot, "Theory of deformation of a porous viscoelastic anisotropic solid," Journal of Applied Physics, vol. 27, pp. 459467, 1956.

[4] M. A. Biot, Mechanics of Incremental Deformation, John Wiley \& Sons, New York, NY, USA, 1965.

[5] J. Pal and A. P. Ghorai, "Propagation of love wave in sandy layer under initial stress above anisotropic porous half-space under gravity," Transport in Porous Media, vol. 109, no. 2, pp. 297-316, 2015.

[6] S. M. Abo-Dahab, K. Lotfy, and A. Gohaly, "Rotation and magnetic field effect on surface waves propagation in an elastic layer lying over a generalized thermoelastic diffusive halfspace with imperfect boundary," Mathematical Problems in Engineering, vol. 2015, Article ID 671783, 15 pages, 2015.

[7] S. M. Ahmed and S. M. Abo-Dahab, "Propagation of Love waves in an orthotropic granular layer under initial stress overlying a semi-infinite granular medium," Journal of Vibration and Control, vol. 16, no. 12, pp. 1845-1858, 2010.

[8] R. Chattaraj and S. K. Samal, "Love waves in the fiber-reinforced layer over a gravitating porous half-space," Acta Geophysica, vol. 61, no. 5, pp. 1170-1183, 2013.

[9] W. Chen, T. Xia, and W. Hu, "A mixture theory analysis for the surface-wave propagation in an unsaturated porous medium," International Journal of Solids and Structures, vol. 48, no. 16-17, pp. 2402-2412, 2011.

[10] V. K. Kalyani, A. Sinha, Pallavika, S. K. Chakraborty, and N. C. Mahanti, "Finite difference modeling of seismic wave propagation in monoclinic media," Acta Geophysica, vol. 56, no. 4, pp. 1074-1089, 2008.

[11] A. P. Ghorai, S. K. Samal, and N. C. Mahanti, "Love waves in a fluid-saturated porous layer under a rigid boundary and lying over an elastic half-space under gravity," Applied Mathematical Modelling, vol. 34, no. 7, pp. 1873-1883, 2010.

[12] A. Chattopadhyay, S. Gupta, P. Kumari, and V. K. Sharma, "Effect of point source and heterogeneity on the propagation of SH-Waves in a viscoelastic layer over a viscoelastic half space," Acta Geophysica, vol. 60, no. 1, pp. 119-139, 2012.

[13] S. Gupta, S. K. Vishwakarma, D. K . Majhi, and S. Kundu, "Possibility of Love wave propagation in a porous layer under the effect of linearly varying directional rigidities," Applied Mathematical Modelling, vol. 37, no. 10-11, pp. 6652-6660, 2013.

[14] S. Gupta, A. Chattopadhyay, and D. K. Majhi, "Effect of initial stress on propagation of love waves in an anisotropic porous layer," Journal of Solid Mechanics, vol. 2, no. 1, pp. 50-62, 2010.

[15] L.-L. Ke, Y.-S. Wang, and Z.-M. Zhang, "Propagation of love waves in an inhomogeneous fluid saturated porous layered half-space with properties varying exponentially," Journal of Engineering Mechanics, vol. 131, no. 12, pp. 1322-1328, 2005.

[16] Z. Kończak, "The propagation of Love waves in a fluid-saturated porous anisotropic layer," Acta Mechanica, vol. 79, no. 3-4, pp. 155-168, 1989.

[17] R. Kumar and R. Kumar, "Analysis of wave motion at the boundary surface of orthotropic thermoelastic material with 
voids and isotropic elastic half-space," Journal of Engineering Physics and Thermophysics, vol. 84, no. 2, pp. 463-478, 2011.

[18] Z. Liu and R. De Boer, "Dispersion and attenuation of surface waves in a fluid-saturated porous medium," Transport in Porous Media, vol. 29, no. 2, pp. 207-223, 1997.

[19] S. K. Chakraborty and S. Dey, "The propagation of Love waves in water-saturated soil underlain by a heterogeneous elastic medium," Acta Mechanica, vol. 44, no. 3-4, pp. 169-176, 1982.

[20] M. D. Sharma, "Wave propagation in a general anisotropic poroelastic medium with anisotropic permeability: phase velocity and attenuation," International Journal of Solids and Structures, vol. 41, no. 16-17, pp. 4587-4597, 2004.

[21] Y.-S. Wang and Z.-M. Zhang, "Propagation of Love waves in a transversely isotropic fluid-saturated porous layered halfspace," Journal of the Acoustical Society of America, vol. 103, no. 2, pp. 695-701, 1998.

[22] A. M. Abd-Alla, S. M. Abo-Dahab, and T. A. Al-Thamali, "Love waves in a non-homogeneous orthotropic magneto-elastic layer under initial stress overlying a semi-infinite medium," Journal of Computational and Theoretical Nanoscience, vol. 10, no. 1, pp. 10-18, 2013.

[23] A. M. Abd-Alla, A. Khan, and S. M. Abo-Dahab, "Rotational effect on Rayleigh, Love and Stoneley waves in fibre-reinforced anisotropic general viscoelastic media of higher and fraction orders with voids," Journal of Mechanical Science and Technology, vol. 29, no. 10, pp. 4289-4297, 2015.

[24] A. M. Abd-Alla, H. A. Hammad, and S. M. Abo-Dahab, "Rayleigh waves in a magnetoelastic half-space of orthotropic material under influence of initial stress and gravity field," Applied Mathematics and Computation, vol. 154, no. 2, pp. 583597, 2004.

[25] A. M. Abd-Alla, S. R. Mahmoud, S. M. Abo-Dahab, and M. I. Helmy, "Influences of rotation, magnetic field, initial stress, and gravity on Rayleigh waves in a homogeneous orthotropic elastic half-space," Applied Mathematical Sciences, vol. 4, no. 2, pp. 91$108,2010$.

[26] A. M. Abd-Alla, S. M. Abo-Dahab, H. A. Hammad, and S. R. Mahmoud, "On generalized magneto-thermoelastic Rayleigh waves in a granular medium under the influence of a gravity field and initial stress," Journal of Vibration and Control, vol. 17, no. 1, pp. 115-128, 2011.

[27] A. N. Abd-alla and S. M. Abo-dahab, "Rayleigh waves in magneto-thermo-viscoelastic solid with thermal relaxation times," Applied Mathematics and Computation, vol. 149, no. 3, pp. 861-877, 2004.

[28] S. M. Abo-Dahab, A. M. Abd-Alla, and A. Khan, "Magnetism and rotation effect on surface waves in fibre-reinforced anisotropic general viscoelastic media of higher order," Journal of Mechanical Science and Technology, vol. 29, no. 8, pp. 33813394, 2015.

[29] A. E. H. Love, Some Problems of Geodynamics, Cambridge University Press, Cambridge, UK, 1911.

[30] D. Gubbins, Seismology and Plate Tectonics, Cambridge University Press, Cambridge, UK, 1990. 


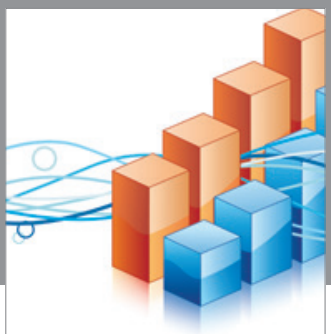

Advances in

Operations Research

vatem alat4

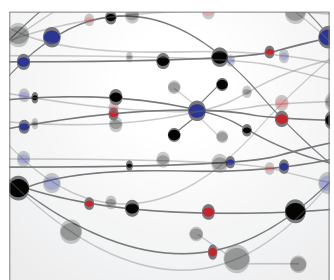

\section{The Scientific} World Journal
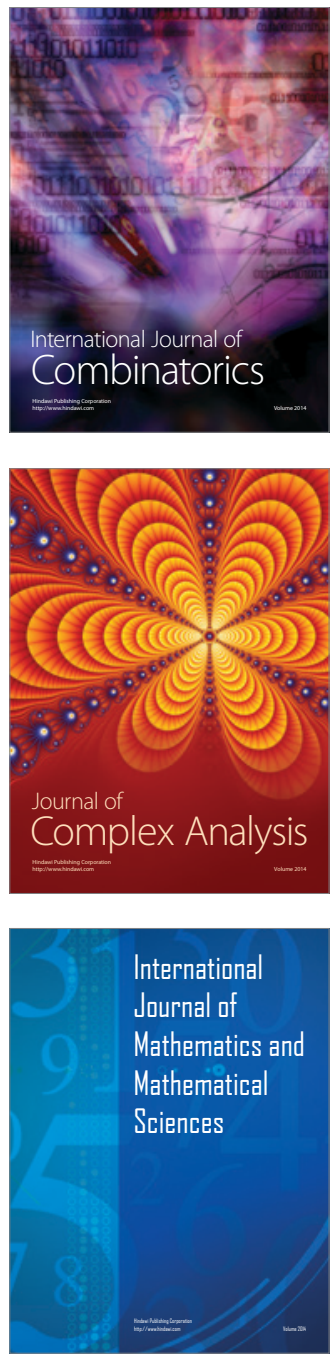
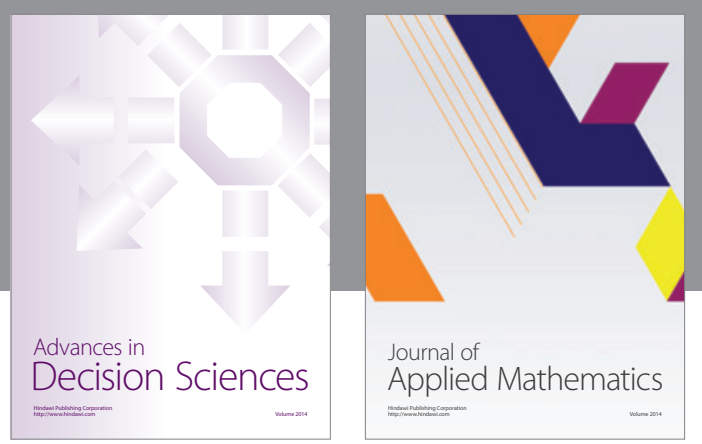

Algebra

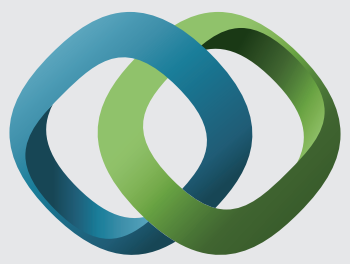

\section{Hindawi}

Submit your manuscripts at

http://www.hindawi.com
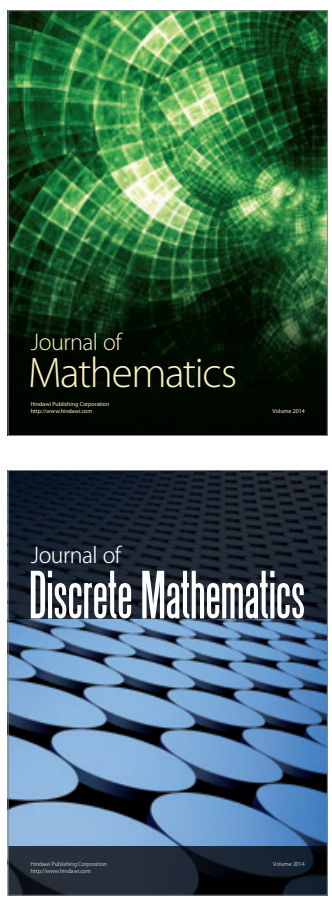

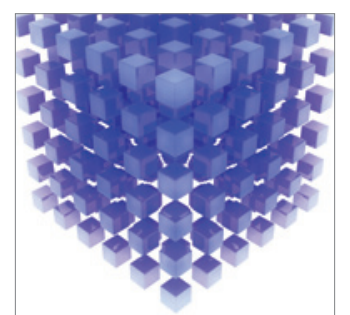

Mathematical Problems in Engineering
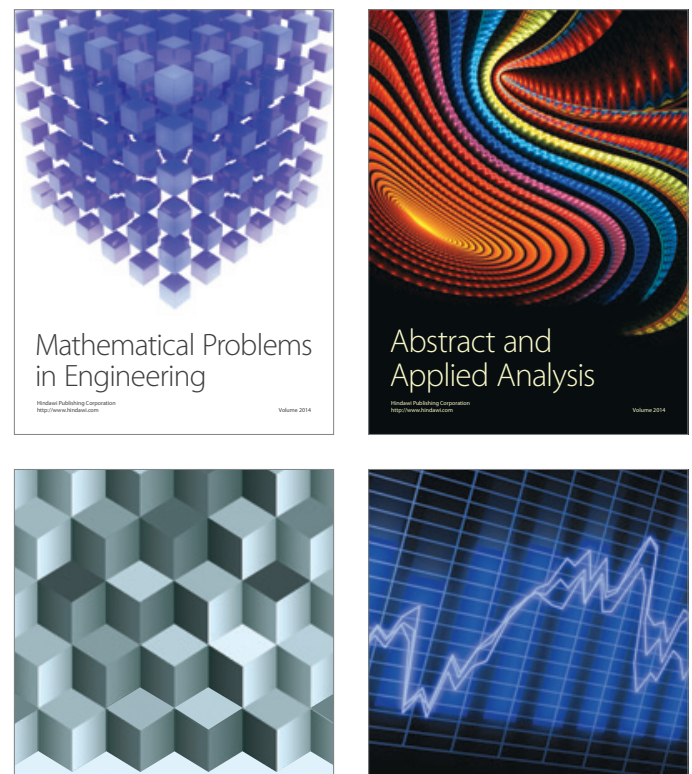

Journal of

Function Spaces

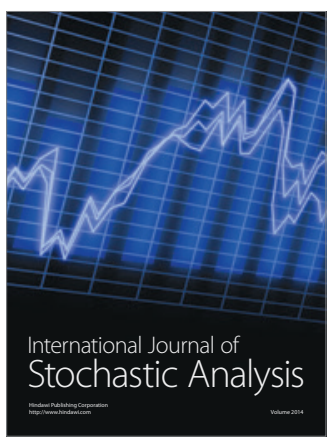

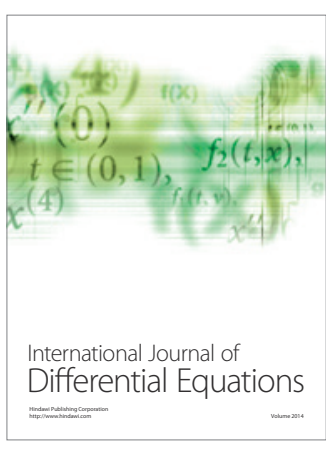
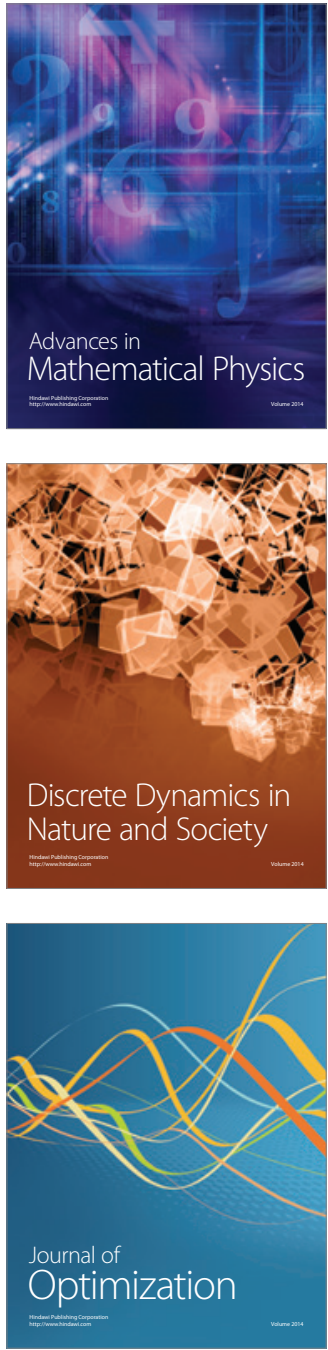